\title{
Aspiração de Corpo Estranho no Idoso
}

\section{Foreign Body Aspiration in the Elderly}

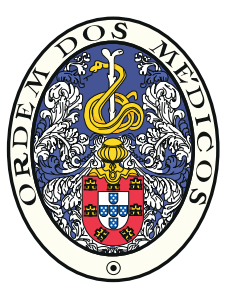

Cátia MATOS $\triangle^{1}$, Manuel Mário SOUSA ${ }^{1}$

Acta Med Port 2016 May;29(5):340-343 - http://dx.doi.org/10.20344/amp.6986

RESUMO

A aspiração de corpo estranho é mais frequente em crianças e idosos, podendo ter uma apresentação subtil neste último grupo. A suspeição clínica é crucial, sendo a broncoscopia o principal meio de diagnóstico e tratamento disponível. Descreve-se o caso de um doente do sexo masculino, 78 anos, antecedentes de doença pulmonar obstrutiva crónica, com quadro de dificuldade respiratória após toma de comprimido, tendo sido levantada a suspeita de aspiração de corpo estranho através da anamnese e achados ao exame objectivo. O diagnóstico foi confirmado através de broncofibroscopia flexível e o tratamento através de broncoscopia rígida. Actualmente, encontra-se estável e com disfagia em estudo.

Palavras-chave: Aspiração Respiratória; Broncoscopia; Corpo Estranho; Idoso; Obstrução da Via Aérea.

\section{ABSTRACT}

Foreign body aspiration is common in children and in the elderly, who may present with subtle symptoms. Clinical suspicion is crucial and bronchoscopy is the main diagnostic and therapeutic procedure available. This is the case of a man, 78 years old, with a history of chronic obstructive pulmonary disease, who presented with respiratory distress following oral intake of tablets. History taking and physical examination raised suspicion. The diagnosis was confirmed with flexible bronchoscopy and rigid bronchoscopy was carried out for treatment. The patient's condition is stable and he is under investigations for dysphagia.

Keywords: Aged; Airway Obstruction; Bronchoscopy; Foreign Bodies; Respiratory Aspiration.

\section{INTRODUÇÃo}

A aspiração de corpos estranhos (ACE) é mais comum em crianças e idosos, ocorrendo abaixo dos 15 anos em aproximadamente $80 \%$ dos casos. Os principais factores de risco na idade adulta são a idade acima de 75 anos, distúrbios neurológicos e psiquiátricos, alterações de consciência por diferentes motivos (como, abuso de ingestão alcoólica e/ou de benzodiazepinas) ${ }^{1}$ e mastigação ineficiente pelo uso de próteses dentárias inadequadas. ${ }^{2}$

A ACE nos adultos pode apresentar-se com quadro clínico subtil ou silencioso, devido à obstrução frequente a nível dos brônquios lobares inferiores ou intermédios. É necessário um elevado grau de suspeição clínica e um exame objetivo atento para o diagnóstico. A tosse é o sintoma mais comum, surgindo em cerca de $80 \%$ dos $\operatorname{casos}^{1}$ e a dispneia surge em apenas $25 \% .^{3}$ Febre, hemoptises, dor torácica, asma resistente ao tratamento ou pneumonias de repetição podem também surgir, sendo muitas vezes, neste contexto que é realizada a broncoscopia que identifica o corpo estranho. ${ }^{4}$

O atraso no diagnóstico pode conduzir a diversas complicações, tais como, pneumonia de aspiração, abcesso pulmonar, hemoptises recorrentes, bronquiectasias e morte. ${ }^{5}$

A hiperinsuflação unilateral pode ser observada na telerradiografia do tórax, principalmente em crianças. ${ }^{6}$ Outros achados radiológicos em adultos, poderão ser áreas de consolidação, derrame pleural e corpo estranho radiopaco (na maioria, peças dentárias) visível, frequentemente, ao nível do brônquio principal direito. ${ }^{6,7}$ Contudo, em $20 \%$ dos casos confirmados de ACE, as telerradiografias do tórax

\section{são normais. ${ }^{8}$}

A tomografia computorizada é mais sensível e específica do que a telerradiografia torácica e os sinais encontrados, quer directos - visualização de corpo estranho, particularmente radiopacos -, quer indirectos - atelectasias, hiperlucência por "air-trapping", bronquiectasias, consolidação lobar e derrame pleural, ${ }^{9}$ podem auxiliar no diagnóstico, contudo, acarretam mais custos, exposição a radiação e, independentemente do resultado, não dispensam a realização de broncoscopia. ${ }^{10,11} \mathrm{~A}$ ressonância magnética, fluoroscopia e cintilografia não têm papel diagnóstico nos dias de hoje. ${ }^{10,11}$

Em doentes estáveis, a broncofibroscopia deve ser a primeira opção diagnóstica, uma vez que possibilita a identificação e localização precisas do corpo estranho e permite a sua extracção das vias aéreas distais. ${ }^{2}$ Contudo, quando esta falha, por exemplo no caso de o corpo estranho estar impregnado com tecido de granulação ou quando há hemoptises importantes, deve ser feita a broncoscopia rígida. Não existe consenso quanto ao tipo de broncoscopia ideal para extração, ${ }^{5}$ pelo que o broncoscopista deve dominar as duas técnicas para ser capaz de converter imediatamente o tipo de procedimento, sempre que necessário., ${ }^{2,12}$

\section{CASO CLÍNICO}

Doente do sexo masculino, 78 anos, caucasiano, viúvo, autónomo nas actividades de vida diária, vive com o neto de 15 anos. Antecedentes pessoais de hipertensão arterial essencial, doença pulmonar obstrutiva crónica GOLD 2 e categoria $\mathrm{B},{ }^{13}$ bronquiectasias sequelares a

\footnotetext{
1. Departamento de Medicina Geral e Familiar. Unidade de Saúde Familiar de Espinho. Espinho. Portugal.

$\square$ Autor correspondente: Cátia Matos. catia.oliveiramatos@gmail.com

Recebido: 10 de setembro de 2015 - Aceite: 28 de janeiro de 2016| Copyright @ Ordem dos Médicos 2016
} 


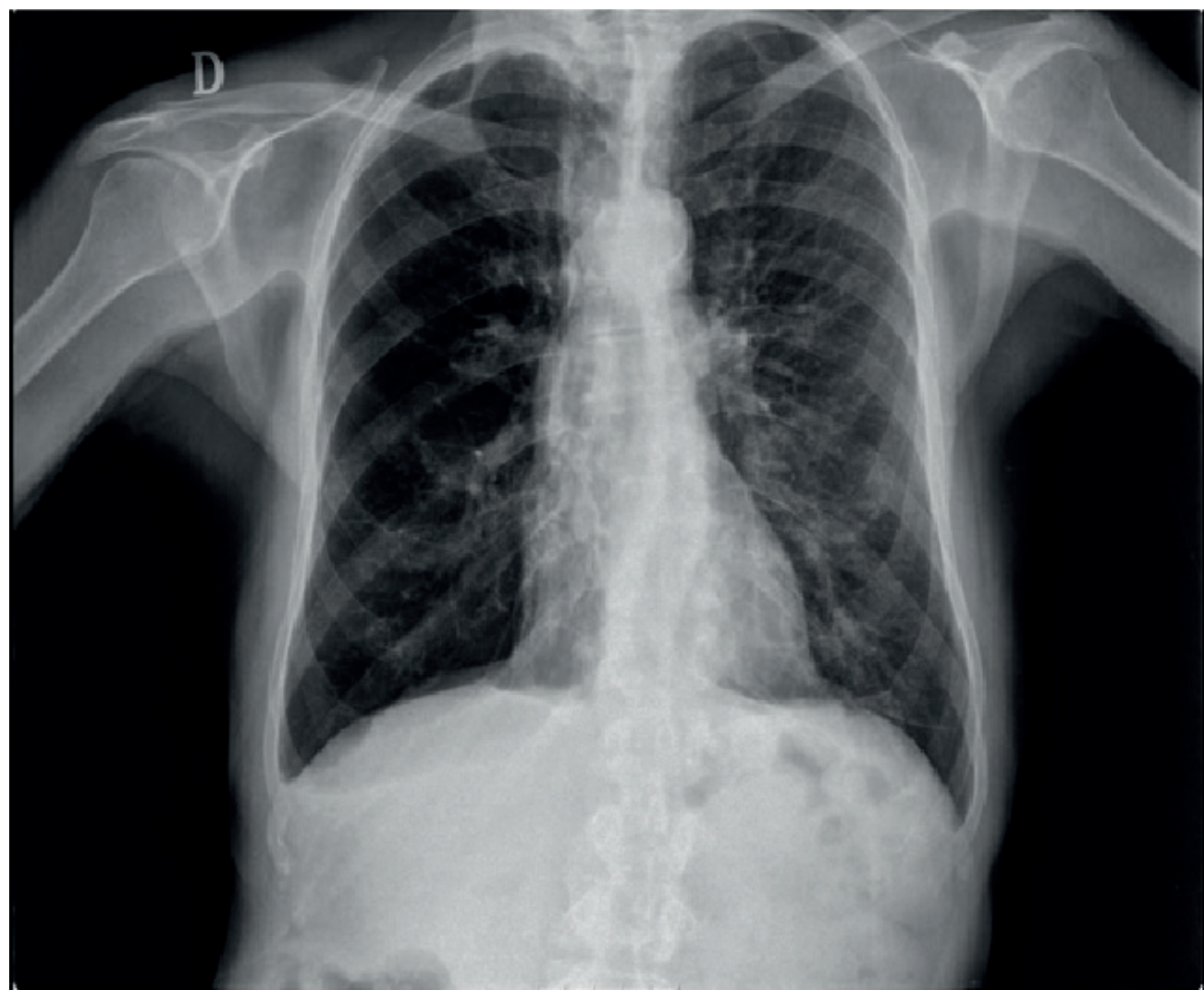

Figura 1 - Telerradiografia do tórax incidência póstero-anterior

tuberculose pulmonar na adolescência e úlcera crónica no membro inferior direito. Sem hábitos tabágicos e/ou etílicos e sem alergias medicamentosas conhecidas.

O doente iniciara antibioterapia com ciprofloxacina e metronidazol por úlcera infectada por Pseudomonas aeruginosa, tendo recorrido a nova consulta três dias depois com queixas de dispneia com início na noite anterior, após toma dos antibióticos, referindo "engasgamento", mas com noção de expulsão total dos comprimidos.

Apresentava sinais de dificuldade respiratória evi-

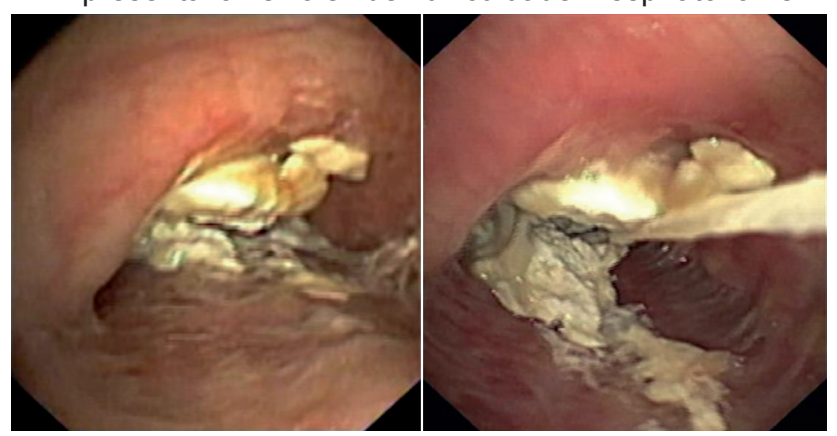

Figura 2 - Visualização do brônquio principal esquerdo com corpo estranho e secreções mucopurulentas, através de videobroncofibroscopia dentes, com taquipneia (36 cpm), pieira audível, tiragem subcostal e cianose periférica. Encontrava-se apirético e com saturação de oxigénio periférico, em ar ambiente, de $96 \%$. À auscultação pulmonar, apresentava uma diminuição franca do murmúrio vesicular em todo o hemitórax direito, com sibilância bilateral. Foi feita nebulização com broncodilatador e pedida telerradiografia torácica urgente, que mostrou hipertransparência de todo o pulmão direito, com aumento dos espaços intercostais (Fig.1).

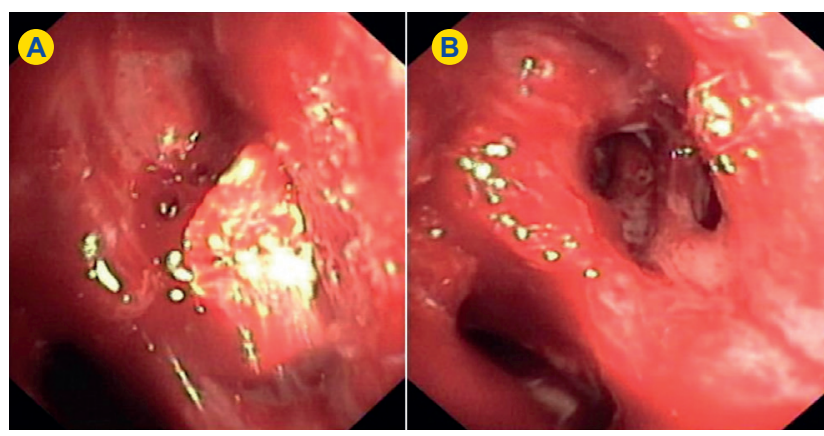

Figura 3 - Visualização do lobo superior esquerdo, carena, lobo inferior esquerdo antes (A) e após extracção de corpo estranho (B) 


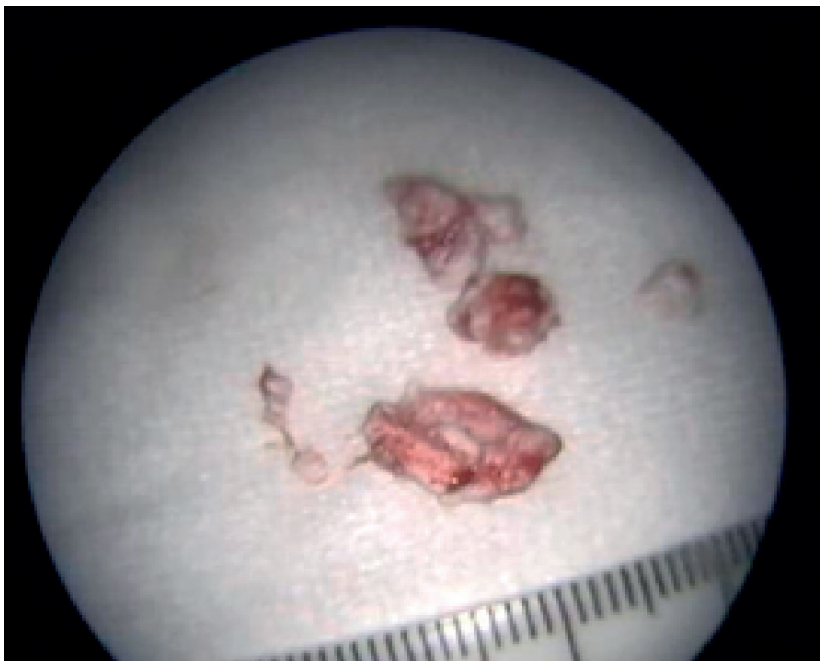

Figura 4 - Alguns dos fragmentos extraídos

Suspeitando de obstrução à esquerda, com hiperinsuflação direita compensatória, o utente foi enviado ao Serviço de Urgência (SU) do hospital de referência. Por manutenção de broncospasmo bilateral refractário, com insuficiência respiratória tipo 1 , realizou broncoscopia que revelou obstrução parcial do brônquio principal esquerdo (BPE) por diversos fragmentos de material envolto em secreções mucopurulentas (Fig. 2), não passíveis de remoção com aspiração ou pinça, pelo que houve necessidade de progressão para broncoscopia rígida.

Foi, então, feita extracção laboriosa com pinça e aspiração de diversos fragmentos de corpo estranho ocupando o BPE e lobo inferior esquerdo, tendo sido obtida repermeabilização completa até ao nível subsegmentar (Figs. 3 e 4).

O estudo bacteriológico e micobacteriológico do lavado brônquico foi negativo. Revisão endoscópica feita duas semanas depois permitiu a extracção completa do corpo estranho ao nível do segmento basal lateral - B9 - esquerdo (Fig. 5). Uma terceira broncofibroscopia comprovou remoção completa do corpo estranho.

\section{DISCUSSÃO}

Apesar da ACE nos adultos ser mais frequente ao nível da árvore brônquica proximal direita, ${ }^{6}$ a aspiração ao nível das vias aéreas inferiores não é rara nos idosos. ${ }^{14}$ A maioria da literatura refere-se a estatística, diagnóstico e tratamento em crianças, sendo a casuística em adultos bastante limitada, variando dos 18 aos 88 anos (média de

\section{REFERENCIAS}

1. Rafanan AL, Mehta AC. Adult airway foreign body removal. What's new? Clin Chest Med. 2001;22:319.

2. Gonçalves MEP, Cardoso SR, Rodrigues AJ. Corpo estranho em via aérea. Pulmão RJ. 2011;20:54-8.

3. Lan RS. Non-asphyxiating tracheobronchial foreign bodies in adults. Eur Respir J. 1994;7:510.

4. Emedicine.medscape.com [homepage na internet]. Foreign body aspiration 2013. [consultado em 1 de novembro de 2015]. Disponível em: http://emedicine.medscape.com/.

5. Bain A, Barthos A, Hoffstein V, Batt J. Foreign-body aspiration in the adult: Presentation and management. Can Respir J. 2013;20:98-9.

6. Pinto A, Scaglione M, Pinto F, Guidi G, Pepe M, Del Prato B, et al. Tracheobronchial aspiration of foreign bodies: current indications for

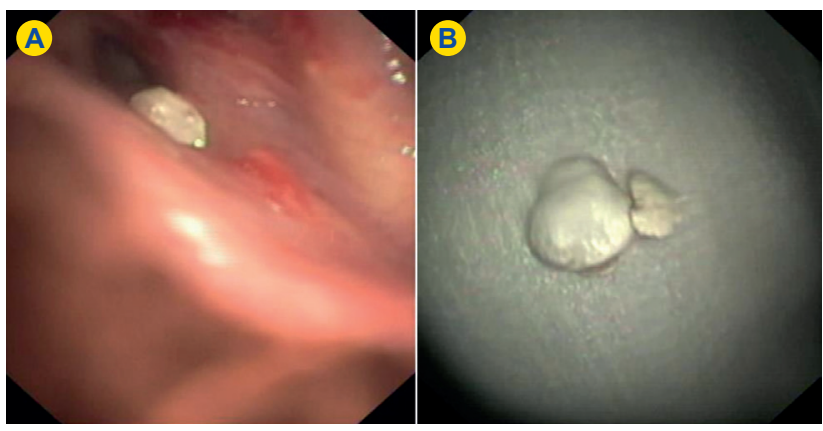

Figura 5 - Corpo estranho no lobo inferior esquerdo visualizado na segunda broncofibroscopia (A); comprimido extraído (B)

60 anos). ${ }^{4}$ Não foi encontrada estatística sobre a aspiração de comprimidos em idosos.

A história de engasgamento foi determinante para a suspeita de ACE. Embora a auscultação pulmonar não fosse típica, sem sibilância unilateral, ${ }^{4}$ as alterações radiológicas e a ausência de melhoria pós-broncodilatação, reforçaram a hipótese inicial.

A broncoscopia flexível possibilitou o diagnóstico, contudo, a broncoscopia rígida, ao facilitar o acesso às vias aéreas subglóticas e a introdução simultânea de múltiplos instrumentos, ${ }^{2,5}$ permitiu uma terapêutica eficaz.

Os autores procuraram realçar, com este caso, a ACE como possível complicação do tratamento farmacológico oral em idosos e os passos necessários para o seu diagnóstico e tratamento atempados.

\section{PROTECÇÃO DE PESSOAS E ANIMAIS}

Os autores declaram que os procedimentos seguidos estavam de acordo com os regulamentos estabelecidos pelos responsáveis da Comissão de Investigação Clínica e Ética e de acordo com a Declaração de Helsínquia da Associação Médica Mundial.

\section{CONFIDENCIALIDADE DOS DADOS}

Os autores declaram ter seguido os protocolos do seu centro de trabalho acerca da publicação dos dados de doentes.

\section{CONFLITOS DE INTERESSE}

Não existem conflitos de interesse a declarar.

\section{FONTES DE FINANCIAMENTO}

Não foi recebido qualquer subsídio ou bolsa.

emergency plain chest radiography. Radiol Med. 2006;111:497-506.

7. Yilmaz A, Akkaya E, Damadoglu E, Gungor S. Occult bronchial foreign body aspiration in adults: analysis of four cases. Respirology. 2004;9:561.

8. Bittencourt PF, Camargos PA, Scheinmann P. Foreign body aspiration: clinical, radiological findings and factors associated with its late removal. Int J Pediatr Otorhinolaryngol. 2006;70:879-84.

9. Zissin R, Shapiro-Feinberg M, Rozenman J, Apter S, Smorjik J, Hertz M. CT findings of the chest in adults with aspirated foreign bodies. Eur Radiol. 2001;11:606.

10. Grover S, Bansal A, Singhi SC. Airway Foreign Body Aspiration. Indian J Pediatr. 2011;78:1401-3.

11. Veras TN, Hornburg G, SchnerAM, Pinto LA. Use of virtual bronchoscopy 
in children with suspected foreign body aspiration. J Bras Penumol. 2009;35:937-41.

12. Moura e Sá J, Oliveira A, Caiado A, Neves S, Barroso A, Almeida J, et al. Tracheobronchial foreign bodies in adults - experience of the Bronchology Unit of Centro Hospitalar de Vila Nova de Gaia. Rev Port Pneumol. 2006;12:31-43.
13. Goldcopd.org [homepage da internet]. Global Strategy for de Diagnosis, Management and Prevention of COPD (updated 2015), Global Initiative for Chronic Obstructive Lung Disease (GOLD); [consultado em 17 de agosto de 2015]. Disponível em: http://www.goldcopd.org/.

14. Teramoto $\mathrm{S}$, Matsuse $\mathrm{T}$, Ouchi $\mathrm{Y}$. Foreign body aspiration into the lower airways may not be unusual in older adults. Chest. 1998;113:1733-4. 